ARTICLE

\title{
Parental relatedness through time revealed by runs of homozygosity in ancient DNA
}

\author{
Harald Ringbauer ${ }^{1,2 \times}$, John Novembre (10 ${ }^{2,3,4} \&$ Matthias Steinrücken $2,3,4$
}

Parental relatedness of present-day humans varies substantially across the globe, but little is known about the past. Here we analyze ancient DNA, leveraging that parental relatedness leaves genomic traces in the form of runs of homozygosity. We present an approach to identify such runs in low-coverage ancient DNA data aided by haplotype information from a modern phased reference panel. Simulation and experiments show that this method robustly detects runs of homozygosity longer than 4 centimorgan for ancient individuals with at least $0.3 \times$ coverage. Analyzing genomic data from 1,785 ancient humans who lived in the last 45,000 years, we detect low rates of first cousin or closer unions across most ancient populations. Moreover, we find a marked decay in background parental relatedness cooccurring with or shortly after the advent of sedentary agriculture. We observe this signal, likely linked to increasing local population sizes, across several geographic transects worldwide.

\footnotetext{
${ }^{1}$ Department of Archaeogenetics, Max Planck Institute for Evolutionary Anthropology, Leipzig, Germany. ${ }^{2}$ Department of Human Genetics, University of Chicago, Chicago, IL, USA. ${ }^{3}$ Department of Ecology and Evolution, University of Chicago, Chicago, IL, USA. ${ }^{4}$ These authors contributed equally: John Novembre, Matthias Steinrücken $凶_{\text {email: harald_ringbauer@eva.mpg.de }}$
} 
A $\mathrm{n}$ individual's parents can be related to each other to varying degrees. For present-day humans, much intriguing geographic variation in parental relatedness has been observed. On one end of the spectrum, globally more than 700 million living humans are the offspring of second cousins or closer relatives. In some regions, the rate of such unions reaches $20-60 \%{ }^{1}$. Parents can also be more distantly related to each other, often via many deeper connections in their pedigree, as a common consequence of small population $\operatorname{sizes}^{2-4}$, or as a consequence of founder effects in tight-knit groups ${ }^{5,6}$. At the other end of the spectrum, in large populations where cousin marriages are not common, many parents have no recent connections in their pedigree at $\mathrm{all}^{2}$. Going back in time, sporadic matings of close kin are documented in royal families of Europe, ancient Egypt, Inca, and pre-contact Hawaii ${ }^{7,8}$, but little is known about broader patterns of past parental relatedness, because archeological evidence alone is typically not informative about mating preferences, especially for prehistoric societies.

The genetic sequence of an individual contains information about the relatedness of their two parents since co-inheritance of identical haplotypes results in stretches of DNA that lack genetic variation (Fig. 1A, often termed runs of homozygosity ${ }^{5}$, though also known by other terms, such as segments with homozygosity by descent (HBD, Supplementary Note 4 ). The more recent the genealogical relationship of the two parents, the more frequent and longer the resulting $\mathrm{ROH}$ tends to $\mathrm{be}^{2}$. $\mathrm{ROH}$ can be identified in genome-wide data ${ }^{9,10}$, and this signal has been analyzed for a wide range of purposes in medical, conservation, and population genetics $^{2}$.

Recently, $\mathrm{ROH}$ has been identified in ancient DNA (aDNA $)^{11-18}$, that is, genetic material extracted from ancient human remains. This advance is especially promising, as large datasets of aDNA have been generated in the last decade ${ }^{19}$.
However, major challenges persist, coverage for aDNA is often around or less than $1 \times$ per site (see Fig. S19), and contamination and DNA degradation introduce genotyping errors $^{20}$. As a consequence, $\mathrm{ROH}$ detection is currently only possible for ancient individuals of exceptional high coverage. Recent methodological advances enable identifying $\mathrm{ROH}$ in data with at least $5 \times$ coverage $^{21}$, but this threshold precludes analysis on all but a small fraction of the currently available aDNA record.

Here, we present an approach to detect $\mathrm{ROH}$ that can identify $\mathrm{ROH}$ longer than 4 centimorgan $(\mathrm{cM})$ in individuals with coverage as low as $0.3 \times$. It is designed to perform well for a common type of human aDNA data: Pseudo-haploid genotypes (Fig. 1B), which consist of a single allele call for each diploid site. Such data do not convey homozygote versus heterozygote genotype states directly; however, as we show, one can extract $\mathrm{ROH}$ from such data by leveraging haplotype information from a phased reference panel.

Using this method, we analyze 1785 ancient individuals from the last 45,000 years, a substantial fraction of the published global human aDNA record. We focused on quantifying two domains of parental relatedness: (1) Close-kin unions, measured by the sum of all $\mathrm{ROH}>20 \mathrm{cM}$, denoted as $\mathrm{sROH}>20$; and (2) background relatedness as measured by the sROH $4-8 \mathrm{cM}$. First, we find that matings among first-cousins or closer relatives, though widely practiced today in numerous societies, are generally infrequently observed in aDNA data. Second, we observe decreasing levels of short $\mathrm{ROH}$ across many regions coinciding with or shortly after the local Neolithic transition from foraging to agricultural subsistence strategies. This genomic evidence of reduced background relatedness supports and refines long-held evidence of the Neolithic transition involving a major demographic shift towards increased local population sizes.
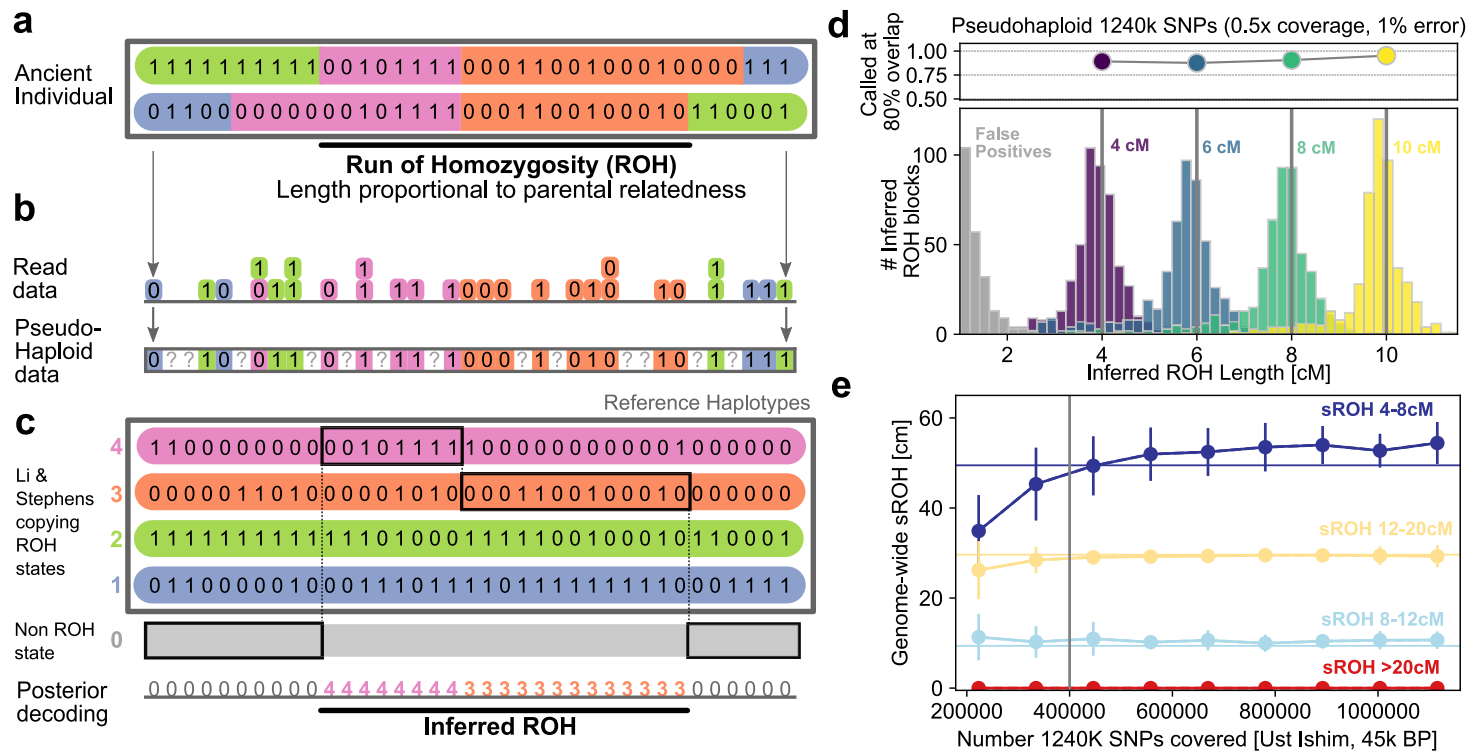

Fig. 1 Detecting runs of homozygosity using a reference panel. a Illustration of genotype data for a diploid individual. $\mathbf{b}$ Mapping sequencing reads to a biallelic SNP produces counts of reads for each allele, from which in turn pseudo-haplotype genotypes, i.e., single reads per site, are sampled. c Schematic of Method. A target individual's genotype data is modeled as mosaic copied from haplotypes in a reference panel (ROH states, colored) and one additional background state (non-ROH, gray). d We applied our method to simulated data with known ROH copied in (see Supplementary Note 1.7 for details). We copied $500 \mathrm{ROH}$ of exactly 4, 6, 8, and $10 \mathrm{cM}$ into 100 artificial chromosomes, and depict histograms of inferred ROH lengths (in color) as well as false positives (in gray) after downsampling and adding errors ( $0.5 \times$ of all $1240 \mathrm{~K}$ SNPs, with $1 \%$ error added). e Down-sampling experiment of a high coverage ancient individual who lived 45,000 years ago (Ust Ishim man) using a modern reference panel (1000 Genomes dataset). We down-sampled pseudohaploid data at the $1240 \mathrm{~K}$ SNPs. For each target coverage, we ran 100 independent replicates and depict the mean and standard deviation of the inferred ROH in four length bins $(4-8,8-12,12-20$, and $>20 \mathrm{cM})$. The horizontal lines indicate high confidence estimates using diploid genotype calls from all available data. 


\section{Results}

Detecting ROH using a haplotype reference panel. Our approach to detect $\mathrm{ROH}$ employs a phased reference panel to leverage haplotype data (described in Supplementary Note 1). Briefly, our method utilizes the fact that sequencing reads in a region of $\mathrm{ROH}$ are effectively sampled from a single haplotype only because the maternal and paternal haplotypes of the diploid individual are identical. In contrast, outside an $\mathrm{ROH}$, two distinct haplotypes are carried by the target individual, and thus, the sequencing reads to originate from both. As a result, modeling sequencing reads as a mosaic of long stretches copied from single reference haplotypes works substantially better within $\mathrm{ROH}$ regions (see Fig. 1). To utilize this signal, we developed a Hidden Markov Model (HMM) with hidden copying states, one for each reference haplotype, to model copying long stretches from the panel [similar to the copying model of ref. ${ }^{22}$ ], and an additional single non- $\mathrm{ROH}$ state as in ref. ${ }^{10}$. We implemented this algorithm in the software package hapROH, available at https:// pypi.org/project/hapROH. The default parameters of the current implementation are tuned for pseudo-haploid genotype calls from a widely used capture technology that targets ca. 1.24 million SNPs [hereafter the "1240K" SNP panel ${ }^{23}$ ] when using a reference panel of 5008 haplotypes of present-day human genetic variation $\left[1000\right.$ Genomes $\left.^{24}\right]$.

Validating the ROH inference. We tested the method in four scenarios: (1) Spiking ROH segments of various lengths (4-10 cM) into data (Supplementary Note 2.1), (2) downsampling high-coverage ancient individuals (Supplementary Note 2.3), (3) down-sampling present-day individuals (Supplementary Note 2.4), and (4) testing different divergence times between the reference panel and the target individual (Supplementary Note 2.2).

For the spike-in experiments, we observe that the power to detect at least $80 \%$ of an inserted $\mathrm{ROH}$ block was above $85 \%$ in all simulated scenarios (Fig. 1D, Supplementary Note 2.1). Bias in the estimated length of the longest overlapping inferred $\mathrm{ROH}$ was consistently below $0.5 \mathrm{cM}$, and we observed no false positives for $\mathrm{ROH}>4 \mathrm{cM}$. In the down-sampling experiments, we tested the ability to recover the sum of $\mathrm{ROH}$ segments falling into four length ranges $(4-8,8-12,12-20$, and $>20 \mathrm{cM})$. When downsampling the oldest modern human genome sequenced to high depth [Ust-Ishim, 45,000 years old ${ }^{25}$ ], the method produces little bias in estimating the respective sROH statistics from pseudohaploid data with as few as 400,000 of the $1240 \mathrm{~K}$ sites covered (see Fig. 1E, Supplementary Note 2.3). In the experiments where we down-sampled 599 present-day individuals from a global sample (Supplementary Note 2.4), the $\mathrm{ROH}$ inference from pseudo-haploid data performs generally well $\left(\mathrm{sROH}_{[4,8]}\right.$ : $r=0.925$ between diploid $\mathrm{ROH}$ calls and pseudo-haploid data, $\mathrm{sROH}_{>20}: r=0.988$, Fig. S10), except for sub-Saharan forager populations. When assessing the impact of different divergence times between the test population and the haplotype reference panel in individuals with a simulated coverage of $1 \times$, we find that using a European-only reference panel, the method can detect $\mathrm{ROH}$ in low coverage test individuals from East Asia and South America but showed much less power for test individuals from West Africa (see Fig. S6).

Together, our tests show that the method can infer $\mathrm{ROH}$ segments longer than $4 \mathrm{cM}$ for individuals with more than 400,000 of the $1240 \mathrm{~K}$ sites covered at least once while tolerating sequencing error rates up to $3 \%$. In addition, our experiments demonstrate that the method can analyze target individuals from populations that diverged from the reference panel up to several ten thousand years ago. Therefore, all present-day and ancient humans that share the out of Africa bottleneck [20,000-40,000 $\mathrm{BP}^{26}$ ] fall into the range of applicability of our method when using the $1240 \mathrm{~K}$ marker set and the full 1000 Genomes dataset as haplotype reference panel.

Application to aDNA data. We then applied our method to a large publicly available dataset of aDNA data (Allen Ancient DNA Resource v42.4, released on March 1, 2020) using the 1000 Genomes dataset as haplotype reference panel (see Section "Methods"). Only 134 of the 3723 individuals in this dataset have average coverage $>5 \times$ (Fig. S19), a typical minimum coverage requirement for previous $\mathrm{ROH}$ methods ${ }^{21}$. Using the method described here with its threshold of 400,000 of the $1240 \mathrm{~K}$ sites covered at least once allowed us to analyze a much larger fraction of this dataset (1833 of the 3723 individuals). We also integrated a dataset of modern individuals genotyped at the Human Origins SNPs $\left[\mathrm{HO}^{27}\right]$, which are a subset of the $1240 \mathrm{~K}$ SNPs. After quality control and filtering (see Section "Methods"), we arrived at a dataset of 1785 ancient and 1941 present-day unique individuals. Within this dataset, we inferred $\mathrm{ROH}$ longer than $4 \mathrm{cM}$ using all available $1240 \mathrm{~K}$ pseudo-haploid data in all ancient individuals and using diploid data for the HO SNPs in all modern individuals. After confirming that $\mathrm{ROH}$ calls on pseudo-haploid and diploid data in modern individuals correlate closely (Pearson correlation coefficient $r=0.925-0.988$, Fig. S8), we analyzed the inferred $\mathrm{ROH}$ in ancient and modern individuals jointly.

Low abundance of long $\mathrm{ROH}$ in ancient humans. We first identified individuals with $\mathrm{sROH}_{>20}$ greater than $50 \mathrm{cM}$. We chose this threshold based on calculations Supplementary Note 4 and simulations Supplementary Note 5, which show that in large populations, $88 \%$ and $20 \%$ of the offspring of first and second cousins, respectively, have $\mathrm{sRH}_{>20}>50 \mathrm{cM}$, but less than $1 \%$ of offspring of third or more distant cousins do. The $50 \mathrm{cM}$ threshold for $\mathrm{sROH}_{>20}$ can also be surpassed in very small isolated populations, specifically, $34 \%$ of individuals in populations of size 250 and $8 \%$ for size 500 (Fig. S15). Hereafter we refer to this as the "long ROH" threshold, and individuals crossing it as having "long $\mathrm{ROH}^{\text {". }}$

Overall, we find that only 54 out of the 1785 ancient individuals $(3.0 \%, \mathrm{CI}: 2.3-3.9 \%)$ have $\mathrm{sROH}_{>20}$ above $50 \mathrm{cM}$. Generally, these individuals with long $\mathrm{ROH}$ do not concentrate in any particular region or time period (Figs. $2 \mathrm{~B}$ and 3 ). The only archeological cluster (defined in annotations from the source dataset, modified for readability) with more than two individuals is "Iron Age Republican Rome", where 3 of 11 samples (reported in ref. ${ }^{28}$ ) fall above the long $\mathrm{ROH}$ threshold. In the PonticCaspian Steppe region, 3 of 54 individuals who lived between 2600 and 1500 BP (5.6\%, CI: 1.2-15.4\%) exceed the threshold (Fig. 2F), but this signal is not significantly different from the rate in the full dataset. Three individuals with long $\mathrm{ROH}$ appear in the late pre-contact Andes region (Fig. 2D), and a follow-up study describes this signal with a larger sample $\operatorname{size}^{29}$. Notably, 11 of the 54 individuals with long $\mathrm{ROH}$ are located on islands: Ordered by time and using the cluster annotations from the publicly available dataset (modified for readability) these are: "Sardinia Early Copper Age" (1 of 1, Fig. S20), "Sweden Megalithic" (1 of 5, all on Gotland), "England Neolithic" (1 of 16), "Chilean Western Archipelago" (1 of 3), "England C-EBA" (2 of 14, Fig. 2), "Russia Bolshoy" (2 of 6), "Vanuatu 1100 BP" (1 of 3), "Argentina Tierra del Fuego" (1 of 1), and "Indian Great Andaman" (1 of 1).

The highest value of $\mathrm{sROH}_{>20}$ across the whole dataset (including present-day individuals) is found in a 6000-year-old Levantine Copper Age individual $\left[11178^{30}\right]$ with $545 \mathrm{cM}$ $\mathrm{sROH}_{>20}$. The other 8 individuals tested from the same burial 

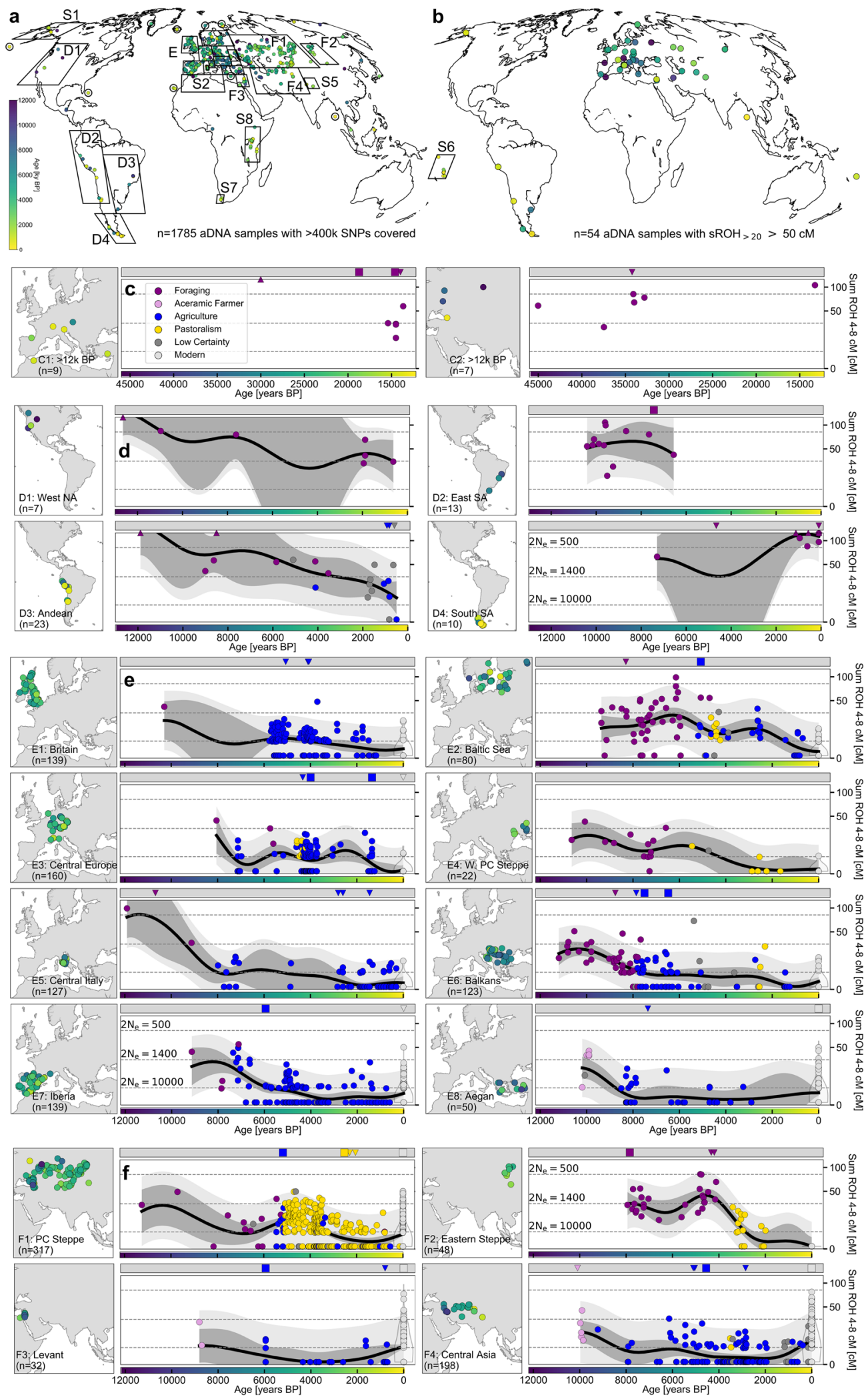

site [Peqi'in Cave, Israel Chalcolithic $6000 \mathrm{BP}^{30}$ ] had $\mathrm{sROH}_{>20}$ values of 0 , and very little $\mathrm{ROH}$ overall $\left(\mathrm{sROH}_{>4}<30 \mathrm{cM}\right)$. The sum and length distribution of $\mathrm{ROH}$ suggest the parents of individual I1178 were first-degree relatives (Fig. 4), i.e., parentoffspring or full siblings whose offspring will have a quarter of their genome in ROH. We note that the burial context of this male individual was not reported to be exceptional.

The rate of long $\mathrm{ROH}$ is substantially higher in the present-day Human Origins dataset; we inferred that 176 of 1941 modern individuals (9.1\%, CI: 7.8-10.4\%) have long ROH. In contrast to 
Fig. 2 Time transects of major regions. a Global distribution of ancient individuals screened for $\mathrm{ROH}$. $\mathbf{b} \mathrm{Global}$ distribution of ancient individuals with long $\mathrm{ROH}$. c-f We plot $\mathbf{s R O H}{ }_{[4,8]}$ for individuals (represented as circles) within several geographic transects (defined in Table S1). Mean estimates were calculated from a Gaussian Process (GP) model (solid black line, see Section "Methods"), as well as 95\% empirical confidence intervals for both individuals (light gray) and for the estimated mean (dark gray). Note the square root scale (chosen for GP modeling, see Section "Methods"). Individuals with values larger than the upper $y$-axis limit are indicated on top of the panels (upward triangles). Horizontal dashed lines depict expectations for $5 \mathrm{ROH}_{[4,8]}$ for panmictic population sizes (see formulas in Supplementary Note 4). In the gray bar at the top of each panel, we indicate individuals with sROH $>20$ more than 100 (squares) and $50 \mathrm{cM}$ (downward triangles), which are plausibly offspring of close kin (held out when fitting the GP). Where available, we show $\mathrm{ROH}$ in present-day individuals (light-gray points for each individual, violin plot for density estimate).

a: Ancient Close Kin Offspring
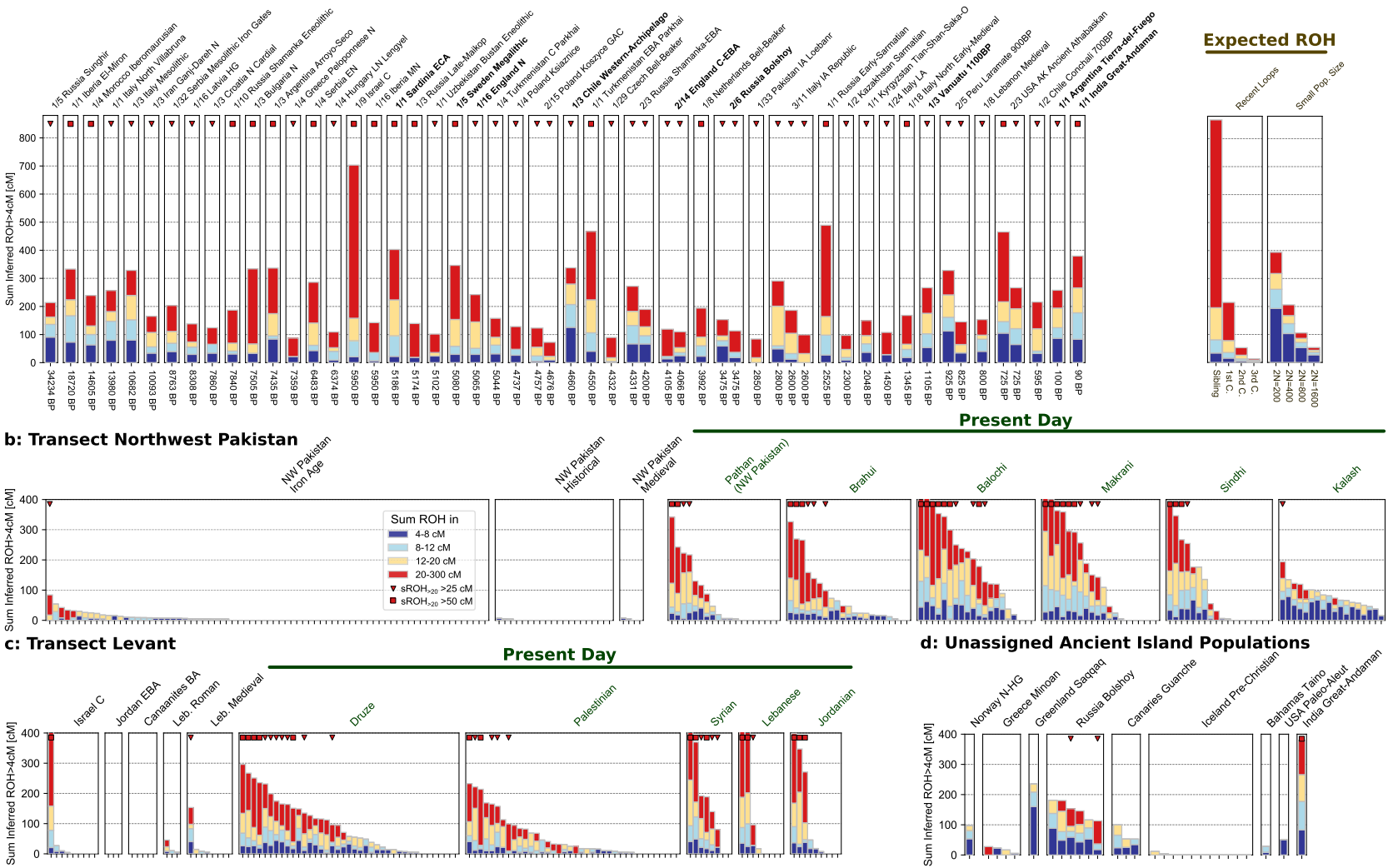

Fig. 3 Individual ROH in a subset of ancient and present-day populations. Each individual is represented by stacked vertical bars, where the length of each bar is determined by the $\mathrm{ROH}$ of this individual falling into four length classes (4-8, 8-12, 12-20, and >20 cM, color-coded). a All 54 ancient individuals (out of 1785) with at least $50 \mathrm{cM} \mathrm{sROH}>20(x / n$ indicates a number of individuals $x$ exceeding the threshold in a cluster of size $n$ defined by archeological label). The labels of the 11 individuals from island populations are highlighted in bold. We also show a legend (top right) of expected ROH for offspring of close kin or in small populations, based on analytical calculations (Supplementary Note 4). For simulations exhibiting individual variation around the mean see Supplementary Note 4 and Supplementary Note 5. b, c Time transects for regions covering present-day Pakistan and Levant, respectively. Modern individuals are indicated by the green horizontal bars. d Ancient individuals from island populations not assigned to geographic regions (circles in Fig. 2a).

ancient data, several geographic clusters of long $\mathrm{ROH}$ are found, mainly in present-day Near East, North Africa, Central/South Asia, and South America (Supplementary Data 1). This signal was described previously [reviewed in ref. ${ }^{2}$ ] and mirrors the estimated prevalence of cousin marriages ${ }^{1}$.

In two regions where long $\mathrm{ROH}$ are common in the presentday data (Fig. 3) our ancient data contains several ancient individuals, which allowed us to analyze time transects. In the Levant, all five present-day annotated groups in our study (Druze, Palestinian, Syrian, Lebanese, Jordanian) have a high fraction of individuals above the long $\mathrm{ROH}$ threshold (30 out of 102 in total, see Fig. 3C). In the ancient sample of this region, only 2 out of 28 analyzed Levant individuals from the Copper Age $(n=9)$, Bronze Age $(n=8)$, Roman times $(n=3)$ to the Middle Age $(n=8)$ fall above this threshold: the first is the Israel Chalcolithic individual with the highest $\mathrm{sROH}_{>20}$ in our dataset (see above) (Fig. 4); the second is a male individual (SI-38) excavated from a mass burial in South Lebanon connected to a Medieval Crusader battle, who was found to have local ancestry ${ }^{31}$. The second region for which we could analyze a time transect is the region of present-day Pakistan. In five out of six modern annotated groups in the dataset (Pathan, Brahui, Makrani, Balochi, and Sindhi), many individuals have long ROH (33 out of 98 individuals with $\mathrm{sROH}_{>20}$ above $50 \mathrm{cM}$ ). In the sixth group, from the Kalash, an isolated valley population, only 1 individual out of 18 exceeds this threshold, despite elevated levels of background $\mathrm{ROH}$ being observed (Fig. 3B). In contrast, in the ancient individuals ${ }^{32}$ [from present-day Northwestern Pakistan], we infer that only 1 

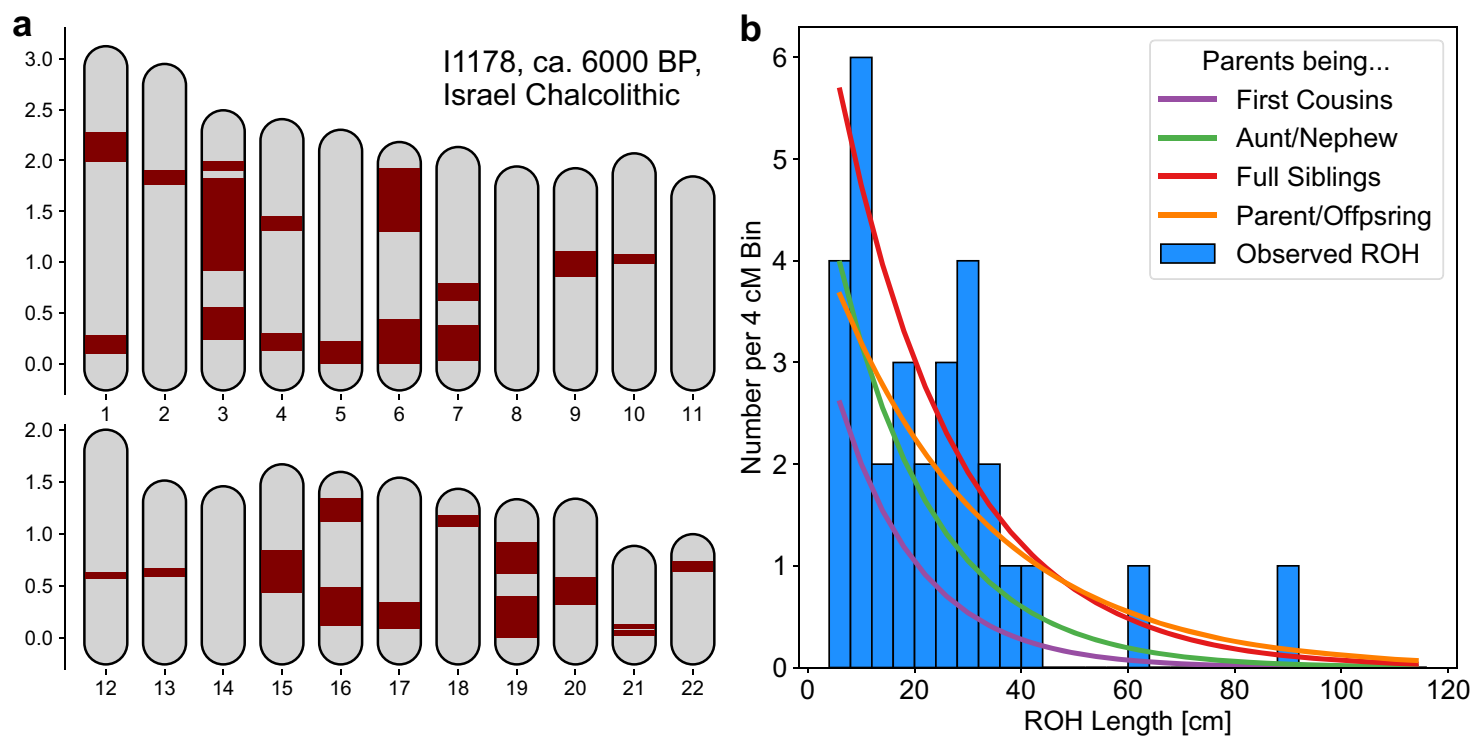

Fig. 4 ROH in a 6000-year-old individual. We show ROH of the individual with the highest sum of inferred ROH among all our samples, I1178, a male individual context-dated to ca. 4500-3900 BCE, reported in ref. ${ }^{30}$. We inferred $703.2 \mathrm{cM}$ of his genome in ROH longer than $4 \mathrm{cM}$, with the longest ROH spanning $91.1 \mathrm{cM}$. a We marked the position of these ROH on the 22 autosomes (maroon), with map length annotated in Morgan. $\mathbf{b}$ We depict a histogram of the $\mathrm{ROH}$ lengths, together with expected densities of $\mathrm{ROH}$ for certain degrees of parental relationships, calculated as described in Supplementary Note 4.

individual out of 75 from the Iron Age (3200-2700 BP) has $\mathrm{sROH}_{>20}$ above the threshold and that none of the 20 individuals from the Historical Period (2600-1900 BP) and none of the 4 individuals from the Middle Period (900-400 BP) surpass the long $\mathrm{ROH}$ threshold (Fig. 3B).

Human background relatedness decreased over time. Shorter $\mathrm{ROH}$ segments measured by $\mathrm{sROH}_{[4,8]}$ accumulate from parental lineages coalescing on average 10-30 generations ago (Fig. S14); thus, their abundance reflects the size of the ancestral mating pool (background relatedness) over approximately the previous half millennium [assuming 30 years per human generation ${ }^{33}$ ]. Because ancestry often spreads out geographically back in time, the probability of recent coalescence and $\mathrm{ROH}$ decreases not only with increasing local population size but also increasing parentoffspring dispersal ${ }^{34,35}$. Assuming that individual mobility is comparable between groups, $\mathrm{sROH}_{[4,8]}$ proxies for local population size ${ }^{36}$. We plotted the values of $\mathrm{sROH}_{[4,8]}$ in time transects for 24 major geographic regions that cover 1763 of the 1785 ancient individuals (16 regions shown in Fig. 2, 8 regions in Fig. S20, 29 additional individuals from islands are shown in Fig. 3D, and the remaining 22 individuals are reported in Supplementary Data 1). In addition, we tested whether $\mathrm{sROH}_{[4,8]}$ differs between subsistence strategies (annotated for most ancient individuals, see Section "Methods") in certain regions (PERMANOVA used for Table 1 and $p$-values in the text below).

We find that $\mathrm{sROH}_{[4,8]}$ is highest among the most ancient individuals in the dataset and then generally decreases going forward in time. Each of 43 ancient individuals in the global sample dated to before $10,000 \mathrm{BP}$ was inferred to have $\mathrm{sROH}_{[4,8]}>0$, with a median value of 54.5 (39 individuals shown in Fig. 2). We then observe a substantial decline in $\mathrm{sROH}_{[4,8]}$ coinciding with the Neolithic transition to sedentary, agricultural lifestyles (Fig. 2). In Western Eurasia, we contrasted individuals from forager cultures to those from early farming cultures (i.e., farming cultures within the first 2000 years after the first annotated "Agriculture" individual per region). We found that $\mathrm{sROH}_{[4,8]}$ decreases substantially in all 8 regional transects which contain both annotated foragers and farmers ( $p$-value $<0.05$ in 7 of these 8 transects), and median $\mathrm{sOH}_{[4,8]}$ values drop from 13 to $66 \mathrm{cM}$ per foraging group to $0-9 \mathrm{cM}$ per early farming group (Tab. 1). In the Andes, where agriculture gradually increased in intensity starting around $5000 \mathrm{BP}$ in a heterogeneous process lasting thousands of years ${ }^{37}$, the median $\mathrm{sROH}_{[4,8]}$ decreases from 55.4 for foragers to 17.9 for agriculturalists $\left(p=1.2 \times 10^{-2}\right.$, Table 1).

Detailed inspection of the transitions from foraging to farming reveals interesting finer-scale dynamics. First, for the earliest western Eurasian farmers not using ceramics yet, who lived $\sim 10,000$ years ago and predate the Neolithic expansions into Europe, we still observe elevated rates of short $\mathrm{ROH}$, with a median $\mathrm{sROH}_{[4,8]}$ of 36.7, 16.4, and 15.2 in Aegean, Levant, and Central Asian aceramic farmers, respectively, which is comparable to values observed for western Eurasian foragers $\left(\mathrm{sROH}_{[4,8]}\right.$ ranging from 13 to $66 \mathrm{cM}$, Table 1). In all three regions there is a subsequent marked drop to ceramic early farmers, with median $\mathrm{sROH}_{[4,8]}$ decreasing substantially to 0,0 , and 4.8 , respectively $\left(p=6.0 \times 10^{-5}, 3.8 \times 10^{-2}\right.$, and $5.4 \times 10^{-2}$, Table 1$)$.

Furthermore, one ceramic early farming group in our sample stands out: Individuals annotated in the original dataset as Iberian Early Neolithic [7400-7000 $\mathrm{BP}^{38}$ ] have median $\mathrm{sROH}_{[4,8]}$ of $32.8 \mathrm{cM}$, which is substantially higher than in other early Eurasian farmers (median $\mathrm{sROH}_{[4,8]}$ : $0-8.7 \mathrm{cM}$, Table 1). However, in Iberian Middle Neolithic farmers (6800-4600 BP) ROH decreases (median $\operatorname{sROH}_{[4,8]}=0, p=1.0 \times 10^{-5}$, Table 1 ) and becomes typical of other early European farmers. As the early Iberian individuals have exceptionally high early farmer ancestry $\left[>90 \%{ }^{38}\right]$, this signal cannot be explained by forager (huntergatherer) ancestry. However, archeological evidence of a rapid maritime spread (Cardial Ware expansion) within a few hundred years around $7500 \mathrm{BP}^{39}$ provides one plausible explanation of this increased abundance of short $\mathrm{ROH}$ in the Early Neolithic, as a rapid spread could have caused an initial bottleneck. Moreover, an initially small population of early farmers would explain why forager admixture substantially increased in Middle Neolithic Iberians and remained one of the highest of European Neolithic populations $\left[\sim 25 \%{ }^{38}\right]$. 
Table 1 Comparison of statistics for $\mathrm{SROH}_{[4,8]}$ for pairs of groups.

\begin{tabular}{|c|c|c|c|c|c|c|c|c|c|c|}
\hline \multirow[b]{2}{*}{ Group A } & \multirow[b]{2}{*}{ Group B } & \multirow[b]{2}{*}{$n_{A}$} & \multirow[b]{2}{*}{$n_{B}$} & \multicolumn{3}{|c|}{$\mathrm{sROH}_{[4,8]} A$} & \multicolumn{3}{|c|}{$\mathrm{sROH}_{[4,8]} \mathrm{B}$} & \multirow[b]{2}{*}{$p$-Value } \\
\hline & & & & $25 \%$ & $50 \%$ & $75 \%$ & $25 \%$ & $50 \%$ & $75 \%$ & \\
\hline $\mathrm{FG}>10 \mathrm{k} \mathrm{BP}$ & FG 8-10k BP & 35 & 53 & 29.2 & 51.0 & 81.6 & 9.9 & 20.8 & 39.2 & $3.5 \times 10^{-4}$ \\
\hline Foraging & Early Farmer & & & & & & & & & \\
\hline All Eurasian & & 111 & 223 & 7.2 & 18.7 & 37.5 & 0.0 & 4.6 & 9.4 & $1.0 \times 10^{-5}$ \\
\hline Aegan & & 1 & 27 & 30.9 & 30.9 & 30.9 & 0.0 & 0.0 & 5.0 & $3.6 \times 10^{-2}$ \\
\hline Balkans & & 38 & 35 & 5.6 & 13.6 & 21.0 & 0.0 & 4.2 & 8.5 & $1.0 \times 10^{-5}$ \\
\hline Baltic Sea & & 41 & 7 & 9.3 & 22.2 & 38.8 & 6.9 & 8.7 & 12.7 & $3.5 \times 10^{-2}$ \\
\hline Britain & & 1 & 90 & 39.8 & 39.8 & 39.8 & 4.2 & 5.6 & 9.4 & $1.1 \times 10^{-2}$ \\
\hline Central Europe & & 4 & 17 & 26.6 & 36.9 & 46.7 & 0.0 & 0.0 & 4.5 & $3.6 \times 10^{-4}$ \\
\hline Iberia & & 4 & 23 & 7.5 & 25.6 & 45.9 & 0.0 & 5.1 & 26.5 & $1.3 \times 10^{-1}$ \\
\hline Central Italy & & 2 & 11 & 49.2 & 65.8 & 82.4 & 0.0 & 4.4 & 10.8 & $1.3 \times 10^{-2}$ \\
\hline Steppe & & 20 & 13 & 5.6 & 17.3 & 51.7 & 0.0 & 4.3 & 9.5 & $9.9 \times 10^{-3}$ \\
\hline Andean & & 7 & 5 & 46.8 & 55.4 & 95.2 & 9.6 & 17.9 & 22.1 & $1.2 \times 10^{-2}$ \\
\hline Farmers $>5 \mathrm{k} \mathrm{BP}$ & Steppe-PA 5.2-3k BP & 286 & 188 & 0.0 & 4.2 & 9.2 & 4.2 & 10.9 & 17.1 & $1.0 \times 10^{-5}$ \\
\hline Central Asian > 3k BP & Steppe-PA 5.2-3k BP & 84 & 188 & 0.0 & 0.0 & 6.2 & 4.2 & 10.9 & 17.1 & $1.0 \times 10^{-5}$ \\
\hline Foraging & Pastoralism & & & & & & & & & \\
\hline Western PC Steppe & & 13 & 8 & 4.5 & 14.2 & 17.3 & 0.0 & 0.0 & 1.0 & $6.9 \times 10^{-3}$ \\
\hline Eastern Steppe & & 28 & 18 & 24.3 & 32.5 & 49.9 & 0.0 & 4.7 & 12.4 & $1.0 \times 10^{-5}$ \\
\hline Aceramic Farmer & Early Ceramic Farmer & & & & & & & & & \\
\hline Aegan & & 5 & 27 & 36.2 & 37.2 & 38.0 & 0.0 & 0.0 & 5.0 & $6.0 \times 10^{-5}$ \\
\hline Levant & & 2 & 11 & 11.0 & 16.4 & 21.8 & 0.0 & 0.0 & 2.0 & $3.8 \times 10^{-2}$ \\
\hline Central Asia & & 5 & 5 & 11.4 & 15.2 & 25.8 & 0.0 & 4.8 & 7.0 & $5.4 \times 10^{-2}$ \\
\hline Iberia-EN & Iberia-MN & 7 & 15 & 23.7 & 32.8 & 37.3 & 0.0 & 0.0 & 4.7 & $1.0 \times 10^{-5}$ \\
\hline Americas & other $<13 \mathrm{k}$ BP & 57 & 1658 & 28.0 & 56.3 & 85.8 & 0.0 & 4.2 & 10.5 & $1.0 \times 10^{-5}$ \\
\hline
\end{tabular}

In the ancient Americas, elevated $\mathrm{sROH}_{[4,8]}$ values evidence sustained high levels of background relatedness. This signal is found across all American regions: Western North America (West NA) (Fig. 2D1, median $\mathrm{sROH}_{[4,8]}=67.1 \mathrm{cM}$ excluding long $\mathrm{ROH}$ individuals, $n=7$ ), Eastern South America (East SA) (Fig. 2D2, $59.0 \mathrm{cM}, n=13$ ), Andean (Fig. 2D3, $31.5 \mathrm{cM}, n=20$ ), Southern SA (Fig. 2D4, $112.5 \mathrm{cM}, n=8$ ) and Beringia (Fig. S20, $52.4 \mathrm{cM}, n=9$ ). This abundance of $\mathrm{ROH}$ (overall median $\left.\mathrm{sROH}_{[4,8]}=56.3\right)$ is higher than the rest of the global sample in the same broad time period $(<13 \mathrm{k}$ years ago, median $4.2 \mathrm{cM}$, $p<10^{-5}$, Table 1). Since $\mathrm{sROH}_{[4,8]}$ is driven by co-ancestry within the last few dozen generations (Supplementary Note 4), this elevated $\mathrm{sROH}_{[4,8]} \mathrm{cM}$ cannot be explained by bottlenecks during early migrations into the Americas, but one needs to invoke more recent, sustained small effective population sizes. Overall we observe little temporal variation (Fig. 2), with one exception in the dataset being Andean populations around the time of the shift to agriculture (see above; also note the dataset does not include individuals from other early centers of agriculture in the Americas, e.g., Central Mexico, eastern North America).

Another observation of elevated $\mathrm{ROH}$ on a large geographical scale is found in the Eurasian Steppe, where early pastoralist groups all have substantial amounts of $\mathrm{sROH}_{[4,8]}$ (Steppe-PA 5.2$3 \mathrm{k} \mathrm{BP}$, median $\mathrm{sROH}_{[4,8]}=10.9$, Table 1$)$, including the Yamnaya (median $17.5 \mathrm{cM}, n=17)$, Afanasievo ( $18.1 \mathrm{cM}, n=22)$, Sintashta $(5.7 \mathrm{cM}, n=21)$, Okunevo $(24.5 \mathrm{cM}, n=12)$ and Srubnaya $(4.8 \mathrm{cM}, n=19)$. These $\mathrm{sROH}_{[4,8]}$ levels are significantly higher than in Western Eurasian farmer populations before $5000 \mathrm{BP}$ (median $4.2 \mathrm{cM}, p=1.0 \times 10^{-5}$, Table 1), and, notably, also significantly higher than their southern contemporaneous neighbors, sedentary farmers from Central Asia (median $0, p=$ $1.0 \times 10^{-5}$, Table 1). In samples from the Western Pontic-
Caspian Steppe (present-day Ukraine and Moldavia), at the transition from foragers to pastoralists, we observe a substantial decrease of $\mathrm{sROH}_{[4,8]}$ from median 14.2 to $0\left(p=6.9 \times 10^{-3}\right.$, Table 1). Similarly in the Eastern Steppe (around Lake Baikal and present-day Mongolia), a shift from foragers to pastoralism coincides with a significant reduction in $\mathrm{sROH}_{[4,8]}$ (median $32.5-4.7, p=1.0 \times 10^{-5}$ ). We note that in both the Western and Eastern Steppe many of the pastoralists in our sample date to 3000-2000 BP (Scythian and Xiongnu, respectively), substantially later than the early pastoralists mentioned above.

\section{Discussion}

We developed a method for measuring $\mathrm{ROH}$ in low coverage ancient DNA. Our algorithm follows a long line of previous work utilizing HMMs to infer such segments ${ }^{10,40-42}$. A key methodological advantage here is to use hidden states that, within an $\mathrm{ROH}$ segment, copy from a reference panel of haplotypes to take advantage of haplotype information. This tool enabled us to screen aDNA data from 1785 individuals for $\mathrm{ROH}$, an order of magnitude more ancient individuals than hitherto amenable for such analysis. We generated evidence for two key aspects of the human past: Identifying long $\mathrm{ROH}(>20 \mathrm{cM})$ provided insight into the past prevalence of close kin unions such as cousin matings, whereas short $\mathrm{ROH}(4-8 \mathrm{cM})$ revealed changing patterns of past background relatedness that reflect local population sizes.

We found that only 1 out of 1785 ancient individuals have long $\mathrm{ROH}$ typical for the offspring of first-degree relatives (e.g., brother-sister or parent-offspring). Historically, matings of firstdegree relatives are only documented in royal families of ancient Egypt, Inca, and pre-contact Hawaii, where they were sporadic occurrences $^{7}$. The only other example of an offspring of firstdegree relatives found using aDNA to date is the recently 
reported case from an elite grave in Neolithic Ireland ${ }^{18}$. Our findings are in agreement that first-degree unions were generally rare in the human past.

Further, we find that only 54 out of 1785 ancient individuals (3.0\%, CI: $2.3-3.9 \%)$ have long $\mathrm{ROH}$ typical for the offspring of first cousins $(88 \%)$ and less commonly observed for second cousins (20\%). Such long ROH can also arise as a consequence of small mating pools (e.g., $8 \%$ in randomly mating populations of size 500, which may explain the long $\mathrm{ROH}$ we observed on certain island populations). Therefore, the rate of long $\mathrm{ROH}$ is an upper bound for the rate of first-cousin unions. On the other hand, because of incomplete power, some long $\mathrm{ROH}$ may be missed in our empirical analysis; however, even if the method would fail to detect half of all $\mathrm{ROH}>20 \mathrm{cM}$, well below the power that we observed in our simulations, we would still detect $60 \%$ of first cousins (see Table S5). We conclude that in our ancient sample substantially less than $10 \%$ of all parental unions occurred on the level of first cousins.

In two specific regions with high levels of long $\mathrm{ROH}$ in the present-day ${ }^{2}$, the dataset contained a sufficient number of ancient individuals to allow analyzing time transects. For both transects (the Levant and present-day Northwest Pakistan), we observe a substantial shift in the levels of long $\mathrm{ROH}$. In contrast to the high abundance of long $\mathrm{ROH}$ typical of close kin unions in the present-day individuals, long $\mathrm{ROH}$ was uncommon in the ancient individuals, including up to the Middle Ages. Additional data from these regions and others with high levels of long $\mathrm{ROH}$ today, such as North Africa as well as Central, South, and West $\mathrm{Asia}^{2}$, will help resolve with more precision the origin and spread of these well-studied kinship-based mating systems ${ }^{43,44}$. Overall, our results show how an $\mathrm{ROH}$-based method can be used to inform understanding of shifts in cultural marriage/mating practices.

As a second major finding, we observed that human background relatedness as measured by short $\mathrm{ROH}(4-8 \mathrm{cM})$ decreased markedly over time in many geographic transects, with a significant drop occurring during or shortly after the local "Neolithic Transition", the transition from a lifestyle of hunting and gathering to one of agriculture and settlement ${ }^{45-47}$. Assuming that early farmers had no increased individual mobility compared to foragers, which would agree with observations in present-day forager populations ${ }^{48}$, the substantial decrease of short $\mathrm{ROH}$ evidences markedly increasing local population sizes. This finding adds support to the long-held hypothesis of local population sizes increasing following the Neolithic transition ${ }^{45-47}$. Previous analysis of ancient genomes of foragers and early farmers already identified several lines of genomic evidence for farmers having larger population sizes than earlier hunter-gatherers, such as decreasing genome-wide diversity ${ }^{49,50}$, decreasing prevalence of $\mathrm{ROH}^{11-14,18}$ and decreasing coalescent rates estimated from high-coverage genomes ${ }^{27}$. Our analysis adds a refined level of geographic and temporal resolution by analyzing an order of magnitude of more individuals (1785 ancient humans) and by organizing those individuals into several densely sampled time transects in different geographic regions.

For individuals from early Eurasian Steppe pastoralist groups, we observe an intermediate level of short ROH. These early cultures (e.g., the Yamnaya) have drawn much attention in archeological and ancient DNA studies to date, as archeological, linguistic, and genetic evidence suggest they played an important role in the origin of Indo-European languages and of several populations expansions ${ }^{32,51-54}$. The elevated rate of short $\mathrm{ROH}$ we observed provides evidence that many matings occurred within and among small, related groups. An alternative interpretation for the abundance of short $\mathrm{ROH}$ could be that burial sites (Kurgans) represent a biased sample of societal classes with more short $\mathrm{ROH}$ than the general populace ${ }^{51}$. However, as short $\mathrm{ROH}$ probes parental ancestry up to several dozen generations into the past, this signal would require reproductive isolation between societal strata maintained over many generations. Therefore, it is likely that at least part of the signal is due to Steppe populations having comparably low population densities or experienced recent bottlenecks.

Our analysis is limited by several caveats. Importantly, skeletal remains accessible by archeological means often do not constitute a random cross-section of past populations. While levels of background relatedness are expected to be similar within a mixing population, rates of close kin unions can vary substantially because of social structure; e.g., elite dynasties may practice close kin unions despite them being uncommon in the general population. Another limitation is the incomplete sampling of the current aDNA record and that for much of the world, we necessarily make inferences from small numbers and sparse sampling. Future work analyzing the rapidly growing ancient DNA record will help to resolve additional details of social and cultural factors operating at finer scales (e.g., leveraging more precise timings of shifts and more subtle shifts in $\mathrm{ROH}$ patterns). In particular, future studies focusing on specific localized questions will increasingly combine archeological and genetic evidence $^{16}$, in ways that will empower the use of the genetic evidence about the past provided by the methodology presented here.

In addition to denser sampling, there are several ways how our analysis can be improved upon by future work. Here we focused our analysis on long $\mathrm{ROH}(>20 \mathrm{cM})$ and short $\mathrm{ROH}(4-8 \mathrm{cM})$. While this dichotomy helped us to disentangle more clearly recent and distant parental relatedness, we expect that future work refining the downstream analysis of $\mathrm{ROH}$ will be able to extract more subtle signatures by looking across all ROH scales. Furthermore, we note that our application focused on a set of SNPs widely used for human ancient DNA (1240K SNPs). For whole-genome sequencing data (available for a subset of the data analyzed here), using all genome-wide variants would likely lower the requirements for coverage below the current limit of 400,000 of the $1240 \mathrm{~K}$ SNPs covered at least once (corresponding to ca. $0.3 \times$ whole-genome sequencing coverage). Another improvement would be using a reference panel that includes ancient haplotypes. Currently, no long-range phased ancient haplotypes are available, but future work will likely produce such data.

One alternative approach to identify $\mathrm{ROH}$ in low coverage ancient genomes could be to use imputation followed by screening for stretches of homozygous markers using standard $\mathrm{ROH}$ detection methods. This was recently done for ancient individuals with $>10 \times$ coverage $^{18}$. Since imputation of genomes was reported to work well to a coverage similar to the low coverage cutoff used here $\left[{ }^{55,56} \mathrm{ca} .0 .5 \times\right]$ and most imputation methods are based on haplotype-copying methods related to the approach utilized here [the $\mathrm{Li}$ and Stephens model ${ }^{22}$, we expect any such approach to perform similar to ours, after appropriate testing and calibration, as conducted for our method. We chose to develop a method utilizing several key advantages of pseudohaploid data, which is more widely available and requires fewer assumptions about genotype quality, making subsequent analysis less prone to batch effects introduced by various isolation, sequencing, and genotyping protocols.

Identifying $\mathrm{ROH}$ can also be a starting point for other powerful applications: $\mathrm{ROH}$ consists of only a single haplotype (the main signal of our method), which is therefore perfectly phased, a prerequisite for powerful methods relying on haplotype copying ${ }^{57}$ or tree reconstruction ${ }^{26,58}$. Moreover, long $\mathrm{ROH}$ could be used to estimate contamination and error rates, an important task in ancient DNA studies ${ }^{20}$. $\mathrm{ROH}$ lacks heterozygotes, allowing one to 
identify heterozygous reads within $\mathrm{ROH}$ that must originate from contamination or genotyping error, similar to estimating contamination from the hemizygous $\mathrm{X}$ chromosomes in males ${ }^{59}$. Another promising future direction is the development of a method to identify long shared sequence blocks in ancient DNA not only within $(\mathrm{ROH})$, but also between individuals, called identity-by-descent (IBD). Calling IBD between individuals would substantially increase power for measuring background relatedness since signals from every pair of individuals could be used. Moreover, a geographic IBD block signal is highly informative about patterns of recent migration ${ }^{35,60-62}$. Extending our method to similarly use haplotype information from a phased reference panel when detecting IBD could enable such analyses in low coverage ancients individuals.

Finally, the analysis of $\mathrm{ROH}$ has additional implications beyond human demography and kinship-based mating systems. In many plants and animal species, $\mathrm{ROH}$ is more prevalent (due to different mating systems, small population sizes, or domestication), and the study of $\mathrm{ROH}$ may be particularly interesting for understanding early plant and animal breeding, as actively controlled mating among domesticates would be expected to alter $\mathrm{ROH}^{63}$. For aDNA from extinct or endangered species, $\mathrm{ROH}$ can shed light on the extinction and inbreeding processes, as is observed for example in aDNA from high-coverage Neanderthal individuals ${ }^{17,64-66}$, or modern DNA from Isle Royal wolves ${ }^{67}$. Finally, as $\mathrm{ROH}$ exposes rare deleterious recessive alleles ${ }^{68}$, the temporal dynamics of $\mathrm{ROH}$ are relevant for understanding the evolutionary dynamics of deleterious variants and health outcomes ${ }^{67,69-71}$. We hope that the core ideas of our approach will inspire the analysis of low-coverage data from a wide range of natural populations.

\section{Methods}

Calling ROH in a global dataset. To detect $\mathrm{ROH}$, we developed a method, hap$\mathrm{ROH}$, which is based on an HMM with ROH and non- $\mathrm{ROH}$ states and uses a panel of reference haplotypes. The detailed method description and evaluation are provided in Supplementary Note 1, Supplementary Note 1.7, Supplementary Note 2.1 and (Supplementary Note 2.4). The software is publicly available at https:// pypi.org/project/hapROH/. Fort the global data analysis we run hapROH (version $0.1 \mathrm{a} 4)$ using the default parameter settings.

Empirical dataset. The global ancient DNA dataset we analyze originates from a curated dataset of published ancient DNA ("1240K", v42.4, released on March 1, 2020, https://reich.hms.harvard.edu). This release provides ancient DNA data in a pseudo-haploid format for 1.24 million SNPs (The 1240K SNP panel). This data includes whole genome as well as $1240 \mathrm{~K}$ SNP capture data compiled from 92 primary publications which were processed starting from bam- or fastq-files using largely identical pipelines across datasets, only adjusting bioinformatics procedures when required by different data generation procedures. We added an additional 40 ancient Sardinian individuals in pseudo-haploid format from a recent publication ${ }^{72}$ that had not yet been compiled into the global reference dataset.

We only analyzed previously generated, publicly available genetic data. For all data, we contacted the corresponding authors of each original study regarding our project and publication plan. We included in our final analysis the data from all studies for which we received a response confirming the use is consistent with the original permits. We filtered to individuals that contained PASS in the ASSESSMENT column of the meta-data table in order to remove individuals with possible contamination. For the remaining ancient individuals that had multiple genotypes listed, we kept the record with the highest coverage. Furthermore, we removed all Neanderthal and Denisovan individuals, as well as the individual tem003, for which initial analysis showed that it has all of chromosome 2 in $\mathrm{ROH}$, but no other long ROH. Finally, we kept only individuals with at least 400,000 SNPs of the 1.24 million covered, the approximate cutoff above which our method can provide robust $\mathrm{ROH}$ inference (Fig. S1). For present-day data, we downloaded the Human Origins dataset with diploid genotype calls for ca. 550,000 autosomal $\mathrm{SNPs}^{27}$, which are a subset of the 1240k SNPs.

We applied hapROH to the pseudo-haploid data for the 1785 ancient individuals and the diploid data for the 1941 modern individuals that pass our quality thresholds. We used all SNPs with available data for which the reference and the alternative allele matched the information in the reference panel, set the respective emission probabilities to values designed for these two types of data (Supplementary Note 1.3), and used the default parameters of hapROH that were optimized for the 1240K SNPs (Supplementary Note 1.8). For the haplotype reference panel, we used the full global set of 5008 phased haplotypes of the 1000 Genomes Project dataset (Phase 3, release 20130502) accessible via http:// ftp.1000genomes.ebi.ac.uk ${ }^{24}$, filtered to biallelic markers and downsampled to SNPs in the 1240K SNP panel with bcftools (version 1.9). This standard human reference dataset is computationally (and in some cases trio-) phased and we kept the phasing as provided. Throughout, we used allele frequencies calculated from the diploid genotypes of the full reference panel when calculating the emission probabilities. We report the detailed ROH calls for all individuals in Supplemental Information 1 .

Annotation of subsistence strategy. For each ancient individual, we annotated the primary subsistence strategy into standard broad categories of food production ${ }^{73}$, using descriptions of the archeological sites and cultural affiliations. We used three main labels: We denoted (1) hunter-gatherer and horticulture lifestyles based on collecting wild plants, hunting, or fishing with the label "Forager"; (2) groups that practiced substantial amounts of sedentary farming (e.g., cereals and domesticates observed in the archeological record) as "Agricultural", and (3) groups with nomadic and semi-nomadic mobile lifestyles based on herding and breeding of domestic animals (e.g., cattle) as "Pastoralist". Groups that had intermediate and transitory lifestyles were annotated using the plausible dominant food economy of the associated archeological culture. To better resolve the transition to agricultural food production, we denoted early groups that practiced agriculture, but lack ceramics in the archeological record as "Aceramic Farmers". Individuals and groups for which the archeological record does not contain sufficient information to annotate a subsistence strategy were labeled as "Uncertain". We stress that archeological evidence is often sparse and assignments are frequently interpretations of various lines of evidence, therefore assessments might change with updates to the archeological record. Here, we tolerate some error, since we address questions regarding very broad temporal and geographic patterns, but we advise against using our subsistence assignments as a reference for questions on a finer scale.

Detecting offspring of close relatives from ROH. We screened all individuals for $\mathrm{ROH}$ longer than $20 \mathrm{cM}$ to identify potential offspring of close relatives. Pairwise IBD $>20 \mathrm{cM}$, which translates to ROH in the offspring, is very unlikely to be a concatenation of multiple shorter IBD blocks ${ }^{74}$. Moreover, recombination quickly breaks up long ancestry segments of the genome, and thus most long ROH originates from co-ancestry within only a small number of generations back. Therefore, if the fraction of the genome in $\mathrm{ROH}$ longer than $20 \mathrm{cM}$ in an individual is large, this provides strong evidence for a close relationship of its parents. We report individuals where the sum of all such $\mathrm{ROH}$ exceeds $50 \mathrm{cM}$ as potential offspring of closely related parents (i.e., $\mathrm{sROH}_{>20}>50$ ). This cutoff is motivated by analytical calculations and simulations, see Supplementary Note 4 and Supplementary Note 5 for details. Briefly, this threshold detects a large fraction of close kin offspring (parents being a first cousin or closer) while also being insensitive to background relatedness unless a population has a very small size $(<500)$

Gaussian process modeling of short ROH. To visualize the trend of the abundance of $\mathrm{ROH}$ in the individuals in certain regions over time, while still conveying the levels of uncertainty due to varying sample sizes, we fit a Gaussian Process (GP) model $^{75}$ using the Python package scikit-learn ${ }^{76}$. As input, we used the square root of the $\mathrm{sROH}_{[4,8]}$ statistic to stabilize its variance ${ }^{77}$, since $\mathrm{sROH}_{[4,8]}$ corresponds closely to count data, which can be approximated by a Poisson distribution. Furthermore, since we use this statistic as a proxy for background relatedness (which in turn proxies for local population size), we removed all individuals with $\mathrm{sROH}_{>20}$ above $50 \mathrm{cM}$ when fitting the GP model, to minimize the impact of putative offspring of close kin on this analysis (Fig. S13).

For the variance model of the GP, we used a standard squared-exponential covariance kernel summed with a residual white noise kernel. In preliminary analyses, we estimated all parameters of the model via maximum likelihood, but we found that these estimates appeared to over-fit the data for several time transects. Thus, we set custom length scales for the covariance kernel for each transect (1500 for all non-American populations and 2000 for American populations, because they had larger temporal sampling gaps) and only fit the two coefficients of the squared-exponential and white noise kernel. To visualize the final output, we estimated the variance of the predicted mean across a dense set of time points ${ }^{75}$. We estimated the uncertainty of the predicted mean and the uncertainty of each individual point and plotted both as $95 \%$ confidence interval bands ( \pm 1.96 standard deviations) on a dense grid.

Analytical expectations of ROH. To aid interpretation of ROH, we visualize expectations of sROH using formulas describing $\mathrm{ROH}$ of closely related parents in otherwise outbred populations and finite populations without substructure. We derive and state these formulas in a unified framework (Supplementary Note 4). We note that these formulas have been derived previously ${ }^{78,79}$. In addition, we verified these formulas by simulating $\mathrm{ROH}$ for these demographic scenarios and comparing expected sROH values to empirical averages (Supplementary Note 4 and Supplementary Note 5). 
Comparing ROH between groups. To test significant differences in the distributions of the sROH statistics between two groups, we applied the Permutational multivariate analysis of variance method [PERMANOVA ${ }^{80}$, which calculates a pseudo-F statistic and assesses its significance via permutation tests. We used the permanova function implemented in the Python package skbio, and based the distance matrices on absolute differences of individual's $\mathrm{sROH}_{[4,8]}$. For each test, we ran 99,999 permutations (minimal $p$-Value: $p=10^{-5}$ ) and report two-sided $p$-Values. As with the GP modeling, we removed all individuals with $s \mathrm{ROH}_{>20}$ above $50 \mathrm{cM}$ when comparing distributions of $\mathrm{sROH}_{[4,8]}$ between groups.

Reporting summary. Further information on research design is available in the Nature Research Reporting Summary linked to this article.

\section{Data availability}

No new DNA data were generated for this study. The ancient dataset and modern data [Human Origins ${ }^{27}$ ] we analyzed originate from the Allen Ancient DNA Resource (Version V42.4, available via https://reich.hms.harvard.edu), primary publications listed in Supplementary Data 1B. The raw reference panel data that we used (phased haplotypes from the 1000 Genomes dataset) is available at http://ftp.1000genomes.ebi.ac.uk/voll/ftp/ release/20130502/. The ancient and modern data we screened for ROH, as well as the processed reference panel we generated (down-sampled to biallelic SNPs at 1240k sites), are archived at https://doi.org/10.5281/zenodo.4992532. The source data underlying Fig. 2, Fig. 3, and Table 1, i.e., the ROH results on ancient DNA data, are provided in Supplementary Data 1A. Code that generates the data for each figure in the main text and Supplementary Information is listed in Supplementary Data 1C.

\section{Code availability}

The Python package implementing the method is available at the Python Package Index (https://pypi.org/project/hapROH/) and can be installed using pip. The documentation provides example use cases as blueprints for custom applications. Code developed for simulating data, analysis, and data visualization is available at the GitHub repository https://github.com/hringbauer/hapROH. The version used for this work is archived at https://doi.org/10.5281/zenodo.4992416 ${ }^{81}$. For data analysis we used Python (3.7.6) and the Python packages jupyterlab (2.1.2), scipy (1.3.1), pandas (1.1.4), numpy (1.19.4), and scikit-bio (0.5.6). We visualized results using matplotlib (3.1.1) and basemap (1.2.1)

Received: 22 September 2020; Accepted: 21 July 2021;

Published online: 14 September 2021

\section{References}

1. Bittles, A. H. \& Black, M. Consanguinity, human evolution, and complex diseases. Proc. Natl Acad. Sci. USA 107, 1779-1786 (2010).

2. Ceballos, F. C., Joshi, P. K., Clark, D. W., Ramsay, M. \& Wilson, J. F. Runs of homozygosity: windows into population history and trait architecture. Nat. Rev. Genet. 19, 220 (2018).

3. Henn, B. M. et al. Hunter-gatherer genomic diversity suggests a Southern African origin for modern humans. Proc. Natl Acad. Sci. USA 108, 5154-5162 (2011).

4. Mondal, M. et al. Genomic analysis of andamanese provides insights into ancient human migration into asia and adaptation. Nat. Genet. 48, 1066-1070 (2016).

5. Broman, K. W. \& Weber, J. L. Long homozygous chromosomal segments in reference families from the centre d'Etude du polymorphisme humain. Am. J. Hum. Genet. 65, 1493-1500 (1999).

6. Goldschmidt, E., Ronen, A. \& Ronen, I. Changing marriage systems in the jewish communities of israel. Ann. Hum. Genet. 24, 191-204 (1960).

7. Bixler, R. H. Sibling incest in the royal families of Egypt, Peru, and Hawaii. J. Sex Res. 18, 264-281 (1982)

8. Ceballos, F. C. \& Álvarez, G. Royal dynasties as human inbreeding laboratories: the Habsburgs. Heredity 111, 114-121 (2013).

9. Purcell, S. et al. PLINK: a tool set for whole-genome association and population-based linkage analyses. Am. J. Hum. Genet. 81, 559-575 (2007).

10. Narasimhan, V. et al. BCFtools/RoH: a hidden Markov model approach for detecting autozygosity from next-generation sequencing data. Bioinformatics 32, 1749-1751 (2016).

11. Gamba, C. et al. Genome flux and stasis in a five millennium transect of european prehistory. Nat. Commun. 5, 1-9 (2014).

12. Jones, E. R. et al. Upper palaeolithic genomes reveal deep roots of modern eurasians. Nat. Commun. 6, 1-8 (2015)

13. Broushaki, F. et al. Early neolithic genomes from the eastern fertile crescent. Science 353, 499-503 (2016).

14. Sikora, M. et al. Ancient genomes show social and reproductive behavior of early Upper Paleolithic foragers. Science 358, 659-662 (2017).
15. Schroeder, H. et al. Origins and genetic legacies of the Caribbean Taino. Proc Natl Acad. Sci. USA 115, 2341-2346 (2018).

16. Racimo, F., Sikora, M., Vander Linden, M., Schroeder, H. \& Lalueza-Fox, C. Beyond broad strokes: sociocultural insights from the study of ancient genomes. Nat. Rev. Genet. 21, 355-366 (2020).

17. Mafessoni, F. et al. A high-coverage Neandertal genome from Chagyrskaya Cave. Proc. Natl Acad. Sci. 117, 15132-15136 (2020).

18. Cassidy, L. M. et al. A dynastic elite in monumental Neolithic society. Nature 582, 384-388 (2020).

19. Skoglund, P. \& Mathieson, I. Ancient genomics of modern humans: the first decade. Annu. Rev. Genom. Hum. Genet. 19, 381-404 (2018).

20. Furtwängler, A. et al. Ratio of mitochondrial to nuclear DNA affects contamination estimates in ancient DNA analysis. Sci. Rep. 8, 14075 (2018).

21. Renaud, G., Hanghøj, K., Korneliussen, T. S., Willerslev, E. \& Orlando, L. Joint estimates of heterozygosity and runs of homozygosity for modern and ancient samples. Genetics. 212, 587-614 (2019).

22. Li, N. \& Stephens, M. Modeling linkage disequilibrium and identifying recombination hotspots using single-nucleotide polymorphism data. Genetics 165, 2213-2233 (2003).

23. $\mathrm{Fu}, \mathrm{Q}$. et al. An early modern human from Romania with a recent Neanderthal ancestor. Nature 524, 216 (2015).

24. Consortium, G. P. et al. A global reference for human genetic variation. Nature 526, 68-74 (2015)

25. Fu, Q. et al. Genome sequence of a 45,000-year-old modern human from western Siberia. Nature 514, 445-449 (2014).

26. Speidel, L., Forest, M., Shi, S. \& Myers, S. R. A method for genome-wide genealogy estimation for thousands of samples. Nat. Genet. 51, 1321-1329 (2019).

27. Lazaridis, I. et al. Ancient human genomes suggest three ancestral populations for present-day Europeans. Nature 513, 409-413 (2014).

28. Antonio, M. L. et al. Ancient Rome: a genetic crossroads of Europe and the Mediterranean. Science 366, 708-714 (2019).

29. Ringbauer, H., Steinrücken, M., Fehren-Schmitz, L. \& Reich, D. Increased rate of close-kin unions in the central andes in the half millennium before european contact. Curr. Biol. 30, R980 - R981 (2020).

30. Harney, É. et al. Ancient DNA from Chalcolithic Israel reveals the role of population mixture in cultural transformation. Nat. Commun. 9, 1-11 (2018).

31. Haber, M. et al. A transient pulse of genetic admixture from the crusaders in the near east identified from ancient genome sequences. Am. J. Hum. Genet. 104, 977-984 (2019).

32. Narasimhan, V. M. et al. The formation of human populations in South and Central Asia. Science 365, eaat7487 (2019).

33. Fenner, J. N. Cross-cultural estimation of the human generation interval for use in genetics-based population divergence studies. Am. J. Phys. Anthropol. 128, 415-423 (2005).

34. Barton, N. H., Depaulis, F. \& Etheridge, A. M. Neutral evolution in spatially continuous populations. Theor. Popul. Biol. 61, 31-48 (2002).

35. Ringbauer, H., Coop, G. \& Barton, N. H. Inferring recent demography from isolation by distance of long shared sequence blocks. Genetics 205, 1335-1351 (2017).

36. Browning, S. R. et al. Ancestry-specific recent effective population size in the Americas. PLoS Genet. 14, el007385 (2018)

37. Piperno, D. R. \& Fritz, G. J. On the emergence of agriculture in the New World. Curr. Anthropol. 35, 637-643 (1994).

38. Olalde, I. et al. The genomic history of the iberian peninsula over the past 8000 years. Science 363, 1230-1234 (2019).

39. Zilhão, J. Radiocarbon evidence for maritime pioneer colonization at the origins of farming in west Mediterranean Europe. Proc. Natl Acad. Sci. USA 98, 14180-14185 (2001).

40. Leutenegger, A.-L. et al. Estimation of the inbreeding coefficient through use of genomic data. Am. J. Hum. Genet. 73, 516-523 (2003).

41. Auton, A. et al. Global distribution of genomic diversity underscores rich complex history of continental human populations. Genome Res. 19, 795-803 (2009).

42. Vieira, F. G., Albrechtsen, A. \& Nielsen, R. Estimating ibd tracts from low coverage ngs data. Bioinformatics 32, 2096-2102 (2016).

43. King-Irani, L. Kinship, class, and ethnicity. Underst. Contemp. Middle East 2 , 299-334 (2004)

44. Korotayev, A. Parallel-cousin (fbd) marriage, islamization, and arabization. Ethnology. 39, 95-407 (2000).

45. Diamond, J. \& Bellwood, P. Farmers and their languages: the first expansions. Science 300, 597-603 (2003).

46. Ammerman, A. J. \& Cavalli-Sforza, L. L. The Neolithic Transition and the Genetics of Populations in Europe, Vol. 836 (Princeton University Press, 2014).

47. Bacci, M. L. A Concise History of World Population (John Wiley \& Sons, 2017).

48. MacDonald, D. H. \& Hewlett, B. S. Reproductive interests and forager mobility. Curr. Anthropol. 40, 501-524 (1999). 
49. Skoglund, P. et al. Genomic diversity and admixture differs for Stone-Age Scandinavian foragers and farmers. Science 344, 747-750 (2014).

50. Kousathanas, A. et al. Inferring heterozygosity from ancient and low coverage genomes. Genetics 205, 317-332 (2017).

51. Anthony, D. W. The Horse, the Wheel, and Language: How Bronze-age Riders from the Eurasian Steppes Shaped the Modern World (Princeton University Press, 2010).

52. Allentoft, M. E. et al. Population genomics of Bronze Age Eurasia. Nature 522, 167-172 (2015).

53. Haak, W. et al. Massive migration from the steppe was a source for IndoEuropean languages in Europe. Nature 522, 207 (2015).

54. de Barros Damgaard, P. et al. The first horse herders and the impact of earlyBronze Age steppe expansions into Asia. Science. 360, eaar7711 (2018).

55. Hui, R., D’Atanasio, E., Cassidy, L. M., Scheib, C. L. \& Kivisild, T. Evaluating genotype imputation pipeline for ultra-low coverage ancient genomes. Sci. Rep. 10, 1-8 (2020).

56. Rubinacci, S., Ribeiro, D. M., Hofmeister, R. J. \& Delaneau, O. Efficient phasing and imputation of low-coverage sequencing data using large reference panels. Nat. Genet. 53, 120-126 (2021).

57. Lawson, D. J., Hellenthal, G., Myers, S. \& Falush, D. Inference of population structure using dense haplotype data. PLoS Genet. 8, e1002453 (2012).

58. Kelleher, J. et al. Inferring whole-genome histories in large population datasets. Nat. Genet. 51, 1330-1338 (2019).

59. Korneliussen, T. S., Albrechtsen, A. \& Nielsen, R. ANGSD: analysis of next generation sequencing data. BMC Bioinformatics 15, 356 (2014).

60. Ralph, P. \& Coop, G. The geography of recent genetic ancestry across europe. PLoS Biol. 11, el001555 (2013).

61. Palamara, P. F. \& Pe'er, I. Inference of historical migration rates via haplotype sharing. Bioinformatics 29, i180-i188 (2013).

62. Al-Asadi, H., Petkova, D., Stephens, M. \& Novembre, J. Estimating recent migration and population-size surfaces. PLoS Genet. 15, e1007908 (2019).

63. Frantz, L. A., Bradley, D. G., Larson, G. \& Orlando, L. Animal domestication in the era of ancient genomics. Nat. Rev. Genet. 21, 449-460 (2020).

64. Prüfer, K. et al. The complete genome sequence of a neanderthal from the altai mountains. Nature 505, 43-49 (2014).

65. Kuhlwilm, M. et al. Ancient gene flow from early modern humans into eastern neanderthals. Nature 530, 429-433 (2016).

66. Prüfer, K. et al. A high-coverage Neandertal genome from Vindija Cave in Croatia. Science 358, 655-658 (2017)

67. Robinson, J. A. et al. Genomic signatures of extensive inbreeding in isle royale wolves, a population on the threshold of extinction. Sci. $A d v$. 5, eaau0757 (2019).

68. Narasimhan, V. M. et al. Health and population effects of rare gene knockouts in adult humans with related parents. Science 352, 474-477 (2016).

69. Szpiech, Z. A. et al. Ancestry-dependent enrichment of deleterious homozygotes in runs of homozygosity. Am. J. Hum. Genet. 105, 747-762 (2019).

70. Clark, D. W. et al. Associations of autozygosity with a broad range of human phenotypes. Nat. Commun. 10, 1-17 (2019).

71. Walters, R., Millwood, I., Lin, K., Mei, X. \& Chen, Z. Associations of autozygosity with a broad range of human phenotypes. Nat. Commun. 10, 1-17 (2019).

72. Marcus, J. H. et al. Genetic history from the Middle Neolithic to present on the Mediterranean island of Sardinia. Nat. Commun. 11, 1-14 (2020).

73. Haviland, W. A., Prins, H. E., McBride, B. \& Walrath, D. Cultural Anthropology: The Human Challenge (Cengage Learning, 2013).

74. Chiang, C. W., Ralph, P. \& Novembre, J. Conflation of short identity-bydescent segments bias their inferred length distribution. G3 6, 1287-1296 (2016).

75. Williams, C. K. \& Rasmussen, C. E. Gaussian processes for machine learning. Vol. 2 (MIT Press Cambridge, MA, 2006).

76. Pedregosa, F. et al. Scikit-learn: machine learning in Python. J. Mach. Learn. Res. 12, 2825-2830 (2011).

77. McCullagh, P. \& Nelder, J. Generalized Linear Models (Monographs on Statistics and Applied Probability 37). (Chapman Hall, London, 1989).

78. Carmi, S., Wilton, P. R., Wakeley, J. \& Pe'er, I. A renewal theory approach to IBD sharing. Theor. Popul. Biol. 97, 35-48 (2014).
79. Browning, S. R. \& Browning, B. L. Accurate non-parametric estimation of recent effective population size from segments of identity by descent. Am. J. Hum. Genet. 97, 404-418 (2015).

80. Anderson, M. J. A new method for non-parametric multivariate analysis of variance. Austral. Ecol. 26, 32-46 (2001).

81. Ringbauer, H. hringbauer/hapRoh. https://doi.org/10.5281/zenodo.4992416 (2021).

\section{Acknowledgements}

We thank the original study authors for sharing their data publicly, and David Reich and his lab, in particular Shop Mallick, for compiling and making publicly accessible a normalized pseudohaploid compilation of those data. We thank David Anthony and Alissa Mittnik for reviewing parts of the subsistence strategy annotations and for helpful discussions. We thank Arjun Biddanda, Shai Carmi, David Schloen, Lars FehrenSchmitz, Montgomery Slatkin, and Mashaal Sohail for their comments on the paper. Funding for H.R. and J.N. was provided by NIH grant R01HG007089 and R01GM132383 to J.N.

\section{Author contributions}

We annotate author contributions using the CRediT Taxonomy labels (https://casrai.org/ credit/). Where multiple individuals serve in the same role, the degree of contribution is specified as "lead", "equal", or "support". Conceptualization (Design of study)-lead: H.R.; support: J.N. and M.S. Software-lead: H.R.; support: M.S. Formal analysis-H.R. Data curation-H.R.; support: J.N. Writing (original draft preparation)-lead: H.R.; support: J.N. and M.S. Writing (review and editing) -input from all authors. Supervision - equal: J.N. and M.S. Project administration-equal: J.N. and M.S. Funding acquisition -J.N.

\section{Funding}

Open Access funding enabled and organized by Projekt DEAL.

\section{Competing interests}

The authors declare no competing interests.

\section{Additional information}

Supplementary information The online version contains supplementary material available at https://doi.org/10.1038/s41467-021-25289-w.

Correspondence and requests for materials should be addressed to Harald Ringbauer

Peer review information Nature Communications thanks Francisco Ceballos and the other, anonymous, reviewer(s) for their contribution to the peer review of this work. Peer reviewer reports are available.

Reprints and permission information is available at http://www.nature.com/reprints

Publisher's note Springer Nature remains neutral with regard to jurisdictional claims in published maps and institutional affiliations.

Open Access This article is licensed under a Creative Commons Attribution 4.0 International License, which permits use, sharing, adaptation, distribution and reproduction in any medium or format, as long as you give appropriate credit to the original author(s) and the source, provide a link to the Creative Commons license, and indicate if changes were made. The images or other third party material in this article are included in the article's Creative Commons license, unles indicated otherwise in a credit line to the material. If material is not included in the article's Creative Commons license and your intended use is not permitted by statutory regulation or exceeds the permitted use, you will need to obtain permission directly from the copyright holder. To view a copy of this license, visit http://creativecommons.org/ licenses/by/4.0/

(C) The Author(s) 2021 